\title{
34.
}

\section{Zur Theorie der Anziehung und der Wärme.}

(Von Herrn Jacob Amsler, Privatdocenten an der Universität in Zürich.)

Man bezeichne durch $d w$ das Element einer geschlossenen Oberfläche $F$, durch $\alpha, \beta, \gamma$ die Winkel, welche ihre (nach aufsen gerichtete) Normale mit den Coordinaten-Axen bildet. Ferner setze man zur Abkürzung:

$$
\begin{aligned}
\delta \boldsymbol{U} & =\frac{\partial^{2} \boldsymbol{U}}{\partial x^{2}}+\frac{\partial^{2} \boldsymbol{U}}{\partial y^{2}}+\frac{\partial^{2} \boldsymbol{U}}{\partial \boldsymbol{z}^{2}}, \\
{\left[\boldsymbol{U}^{\prime}, \boldsymbol{U}\right] } & =\frac{\partial \boldsymbol{U}^{\prime}}{\partial x} \cdot \frac{\partial \boldsymbol{U}}{\partial x}+\frac{\partial \boldsymbol{U}^{\prime}}{\partial y} \cdot \frac{\partial \boldsymbol{U}}{\partial y}+\frac{\partial \boldsymbol{U}^{\prime}}{\partial z} \cdot \frac{\partial \boldsymbol{U}}{\partial z} \\
\left(\frac{\partial \boldsymbol{U}}{\partial \boldsymbol{n}}\right) & =\frac{\partial \boldsymbol{U}}{\partial x} \cos \alpha+\frac{\partial \boldsymbol{U}}{\partial y} \cos \beta+\frac{\partial \boldsymbol{U}}{\partial z} \cos \gamma
\end{aligned}
$$

Alsdann findet folgende, leicht zu verificirende Gleichung Statt:

$$
\text { A. } \int U^{\prime}\left(\frac{\partial U}{\partial n}\right) d w=\iiint U^{\prime} \delta U d x d y d z+\iiint\left[U^{\prime}, U\right] d x d y d z \text {. }
$$

Die Integrationen erstrecken sich über die Oberfläche $F^{\prime}$ und den davon eingeschlossenen Raum. Die Gröfsen $\boldsymbol{U}^{\prime}$ und $\boldsymbol{U}$ sind ganz willkürlich; nur müssen sie und ihre ersten Differentialquotienten innerhalb der Grenzen der Integration stetige Functionen von $x, y, z$ sein.

Unter denselben Bedingungen, und wenn aufserdem $k_{1}, k_{2}, k_{3}$ stetige Functionen von $x, y, z$ sind, gilt die allgemeine Gleichung

$$
\text { (B.) } \begin{aligned}
& \int \boldsymbol{U}^{\prime}\left(k_{1} \frac{\partial U}{\partial x} \cos \alpha+k_{2} \frac{\partial U}{\partial y} \cos \beta+k_{3} \frac{\partial U}{\partial z} \cos \gamma\right) d w \\
= & \iiint U^{\prime}\left\{\frac{\partial\left(k_{1} \frac{\partial U}{\partial x}\right)}{\partial x}+\frac{\partial\left(k_{2} \frac{\partial U}{\partial y}\right)}{\partial y}+\frac{\partial\left(k_{3} \frac{\partial U}{\partial z}\right)}{\partial z}\right\} d x d y d z \\
+ & \iiint\left\{k_{1} \frac{\partial U^{\prime}}{\partial x} \cdot \frac{\partial U}{\partial y}+k_{2} \frac{\partial U^{\prime}}{\partial y} \cdot \frac{\partial U}{\partial y}+k_{3} \frac{\partial U^{\prime}}{\partial z} \cdot \frac{\partial U}{\partial z}\right\} d x d y, d z .
\end{aligned}
$$

Die Grenzen der Integration sind dieselben, wie bei der $(A$.).

Beide Gleichungen lassen sich leicht verallgemeineris, den Fall, daf's statt einer einzigen geschlossenen und zusammenhangenden Oberfläche $F$, deren mehrere gegeben sind. Man darf nur jedes Integral durch eine Summe von ähnlichen Integralen ersetzen, welche sich respective über die verschie- 
denen Oberflächen und die davon eingeschlossenen Räume erstrecken. Zu den bekannten zahlreichen, wichtigen Anwendungen der Gleichungen ( $A$.) und (B.) wollen wir im Folgenden einige neue hinzufügen.

Liouville (Additions aux connaissances des terns, pour l'an 1845) giebt einen sehr einfachen Beweis des Satzes, dafs die Gleichungen, welche die Bedingungen des electrischen Gleichgewichts ausdrücken, nur einer einzigen Lösung fähig sind. In der Theorie des inducirten Magnetismus giebt es einen ähnlichen Satz. Es seien $\boldsymbol{M}_{1}, \boldsymbol{M}_{2}, \ldots \boldsymbol{M}_{m}$ beliebige, der gegenseitigen Einwirkung ausgesetzte Körper, welche die Fähigkeit haben, unter dem Einflufs magnetischer Kräfte vorübergehend magnetisch zu werden. Es sei $\boldsymbol{V}$ das Potential der festen magnetischen Kräfte, $\boldsymbol{Q}$ als Potential des durch Induction erzeugten Magnetismus. Nach Poisson (Mém. de l'Institut. T. VI et VII.) findet sich $\boldsymbol{Q}$ aus folgender Gleichung:

$$
\text { (1.) } \quad Q=-\sum_{1}^{m} \iiint k_{\lambda}\left[Q_{\lambda}+V_{\lambda}, \frac{1}{\varrho_{\lambda}}\right] d x_{\lambda} d y_{\lambda} d z_{\lambda} \text {. }
$$

Die Integrationen im zweiten Gliede erstrecken sich über die von den Körpern $\boldsymbol{M}_{1}, \boldsymbol{M}_{2}, \ldots \boldsymbol{M}_{m}$ eingenommenen Räume. Zur Abkürzung wurde

$$
\boldsymbol{\rho}_{\lambda}=\gamma^{\prime}\left(\left(x-x_{\lambda}\right)^{2}+\left(y-y_{\lambda}\right)^{2}+\left(z-z_{\lambda}\right)^{2}\right)
$$

gesetzi; $\boldsymbol{Q}_{\lambda}, \boldsymbol{V}_{\lambda}$ sind dieselben Functionen von $\boldsymbol{x}_{\lambda,}, y_{\lambda}, \boldsymbol{z}_{\lambda}$ wie $\boldsymbol{Q}$ und $\boldsymbol{V}$ von $x, y, z$. Der Coëfficient $k$ hangt von dem gröfsern oder geringern Vermögen des Körpers $\boldsymbol{M}$ ab, magnetisch zu werden; man könnte ihn deshalb füglich den magnetischen Inductionscoëfficienten nennen. Die Gröfse $k$ ändert sich im allgemeinen mit $x, y, z$, ist aber immer endlich und positiv. Überdies darf man sie als eine innerhalb jedes der gegebenen Körper stetige Function betrachten. Denn, würde bei einem Körper diese Bedingung nicht erfüllt und wäre $k$ längs einer beliebigen Oberfläche $f$ discontinuirlich, so könnte man den Körper als aus zwei oder mehreren Körpern bestehend betrachten, die sich längs der Oberfläche $f$ berühren. Innerhalb jedes dieser Körper wäre nun $\boldsymbol{k}$ stetig.

Der angekündigte Satz lautet:

„Es giebt nur eine einzige Function $\boldsymbol{Q}$, welche der Bedingungs"gleichung des magnetischen Gleichgewichts (1.) genügt."

Beweis. Man setze, es gebe zwei Functionen $\boldsymbol{Q}$ und $\boldsymbol{Q}^{\prime}$, welche der Gleichung (1.) genügen. Dann mufs 


$$
Q^{\prime}=-\sum_{1}^{m} \iiint k_{\lambda}\left[Q_{\lambda}^{\prime}+V_{\lambda}, \frac{1}{\rho_{\lambda}}\right] d x_{\lambda} d y_{\lambda} d z_{\lambda}
$$

sein. Subtrahirt man diese Gleichung von der (1.) und setzt $W=Q-Q^{\prime}$, so erhält man

$$
W=-\sum_{1}^{m} \iiint k_{\lambda}\left[W_{\lambda}, \frac{1}{\varrho_{\lambda}}\right] d x_{\lambda} d y_{\lambda} d z_{\lambda} \text {. }
$$

Der Satz ist bewiesen, wenn man zeigen kann, dafs $\boldsymbol{W}=0$ die einzige reelle Function ist, welche dieser Gleichung genügt.

Aus (2.) folgt

$$
\frac{\partial W}{\partial x}=-\sum_{1}^{m} \iiint k_{\lambda}\left[W_{\lambda}, \frac{1}{\varrho_{\lambda}}\right] d x_{\lambda} d y_{\lambda} d z_{\lambda},
$$

oder, was Dasselbe ist:

$$
\frac{\partial W}{\partial x}=-\sum_{1}^{m} \iiint k_{\mu}\left[W_{\mu}, \frac{1}{\varrho_{\mu}}\right] d x_{\mu} d y_{\mu} d z_{\mu} .
$$

Multiplicirt man diese beiden Gleichungen mit einander, so erhält man $\left(\frac{\partial W}{\partial x}\right)^{2}$ durch eine Summe von sechsfachen Integralen ausgedrückt. Auf dieselbe Weise bilde man $\left(\frac{\partial W}{\partial y}\right)^{2}$ und $\left(\frac{\partial W}{\partial z}\right)^{2}$ und setze die gefundenen Werthe in den Ausdruck

$$
\begin{aligned}
\boldsymbol{P} & =\iiint[\boldsymbol{W}, \boldsymbol{W}] d x d y d z \\
& =\iiint\left\{\left(\frac{\partial \boldsymbol{W}}{\partial x}\right)^{3}+\left(\frac{\partial \boldsymbol{W}}{\partial y}\right)^{2}+\left(\frac{\partial \boldsymbol{W}}{\partial z}\right)^{2}\right\} d x d y d z .
\end{aligned}
$$

Die Integration erstrecke sich über den Raum einer unendlich grofsen Kugel. Führt man die angedeuteten Multiplicationen unter den Integralzeichen aus, so nimmt $\boldsymbol{P}$, wie leicht zu sehen, folgende Gestalt an:

$$
\text { (4.) } P=\sum_{1}^{m} \iiint k_{\lambda}\left[W_{\lambda}, g\right] d x_{\lambda} d y_{\lambda} d z_{\lambda},
$$

wenn zur Abkürzung

gesetzt wird.

$$
\begin{aligned}
g & =\sum_{1}^{m} \iiint k_{\mu}\left[W_{\mu}, h\right] d x_{\mu} d y_{\mu} d z_{\mu} \text { und } \\
h & =\iiint\left[\frac{1}{\varrho_{\lambda}}, \frac{1}{\varrho_{\mu}}\right] d x d y d z
\end{aligned}
$$

Die Werthe von $h$ und $g$ lassen sich in endlicher Form angeben. Man setze in (A.) $U^{\prime}=\frac{1}{\varrho_{\lambda}}$ und betrachte $U$ als Potential einer unendlich kleinen 
Kugel, deren Mittelpunct die Coordinaten $x_{\mu}, y_{\mu}, z_{\mu}$ hat. Ihre Dichtigkeit sei $=1$, ihr Volumen $=u$. Alsdann erhält man

$$
\int \frac{1}{\varrho_{\lambda}} \frac{\partial U}{\partial n} d w=\iiint \frac{1}{\varrho_{\lambda}} \delta U d x d y d z+\iiint\left[\frac{1}{\varrho_{\lambda}}, U\right] d x d y d z
$$

Nach einem bekannten Satze ist für alle Puncte au/serhalb des kleinen Raums $u, \delta U=0$; dagegen für Puncte innerhalb desselben ist $\delta U=-4 \pi$. Also ergiebt sich

$$
\iiint \frac{1}{\varrho_{2}} \delta U d x d y d z=-4 \pi \iiint \frac{d x d y d z}{\varrho_{\lambda}}=-\frac{4 \pi u}{\varrho_{\lambda, \mu}},
$$

wenn $\varrho_{\lambda, \mu}=\sqrt{ }\left(\left(x_{\lambda}-x_{\mu}\right)^{2}+\left(y_{\lambda}-y_{\mu}\right)^{2}+\left(z_{\lambda}-z_{\mu}\right)^{2}\right)$ ist. In den beiden übrigen 'Integralen kann man $U=\frac{u}{\varrho_{\mu}}$ setzen, welches

giebt.

$$
\int \frac{1}{\varrho_{\lambda}} \frac{\partial \frac{1}{\varrho_{\mu}}}{\partial n} d w+\frac{4 I}{\varrho_{\lambda, \mu}}=\iiint\left[\frac{1}{\varrho_{2}}, \frac{1}{\varrho_{\mu}}\right] d x d y d z
$$

Erwägt man nun, dafs das erste Integral, über die Oberfläche einer unendlich grofsen Kugel ausgedehnt, verschwindet, so folgt

$$
h=\iiint\left[\frac{1}{\varrho_{\lambda}}, \frac{1}{\varrho_{\mu}}\right] d x d y d z=\frac{4 \pi}{\varrho_{2, \mu}} .
$$

Hiemit geht der Werth von $g$ in

$$
g=4 \pi \sum_{1}^{m} \iiint k_{\mu}\left[W_{\mu}, \frac{1}{\varrho 2, \mu}\right] d x_{\mu} d y_{\mu} d s_{\mu}
$$

über. Vergleicht man diesen Ausdruck mit der Gleichung (2.), so zeigt sich, dafs also, wegen (4.),

$$
g=-4 \pi W_{\lambda} \text {, }
$$

$$
\boldsymbol{P}=-4 \pi \sum_{1}^{m} \iiint k_{\lambda}\left[W_{\lambda}, W_{\lambda}\right] d x_{\lambda} d y_{\lambda} d z_{\lambda}
$$

ist. Diese Gleichung, verbunden mit (3.), giebt schliefslich:

$$
\text { (5.) } \begin{aligned}
0 & =\iiint\left\{\left(\frac{\partial W}{\partial x}\right)^{2}+\left(\frac{\partial W}{\partial y}\right)^{2}+\left(\frac{\partial W}{\partial z}\right)^{2}\right\} d x d y d z \\
& +4 \pi \sum_{1}^{m} \iiint k_{\lambda}\left\{\left(\frac{\partial W}{\partial x_{\lambda}}\right)^{2}+\left(\frac{\partial W}{\partial y_{\lambda}}\right)^{2}+\left(\frac{\partial W}{\partial z_{\lambda}}\right)^{2}\right\} d x_{\lambda} d y_{\lambda} d z_{\lambda} .
\end{aligned}
$$

Eine Summe von reellen Quadraten, multiplicirt mit reellen und positiven Gröfsen, kann aber nur verschwinden, wenn jeder Term für sich verschwindet. Also mufs nothwendig

$$
\frac{\partial W}{\partial x}=0, \quad \frac{\partial W}{\partial y}=0, \quad \frac{\partial W}{\partial z}=0
$$


für alle Puncte des Raums sein, und folglich; wegen (2.), auch $W=0$. Also hat man identisch $\boldsymbol{Q}=\boldsymbol{Q}^{\prime}$; was zu beweisen war.

Übrigens sieht man leicht, dafs auch kein imaginärer, von 0 verschiedener Ausdruck für $\boldsymbol{W}$ der Gleichung (2.) genügt. Es sei nämlich $\boldsymbol{W}=\boldsymbol{W}^{\prime}+i \boldsymbol{W}^{\prime \prime}$, wo $W^{\prime}$ und $W^{\prime \prime}$ reell sind, so zieht man aus der Gleichung (2.):

$$
\begin{aligned}
& \boldsymbol{W}^{\prime}=-\sum_{1}^{m} \iiint k_{\lambda}\left[W_{\lambda}^{\prime}, \frac{1}{\varrho_{\lambda}}\right] d x_{\lambda} d y_{\lambda} d z_{\lambda} \text { und } \\
& \boldsymbol{W}^{\prime \prime}=-\sum_{1}^{m} \iiint k_{\lambda}\left[W_{\lambda}^{\prime \prime}, \frac{1}{\varrho_{\lambda}}\right] d x_{\lambda} d y_{\lambda} d z_{\lambda,},
\end{aligned}
$$

woraus, wie vorher, $W^{\prime}=0, W^{\prime \prime}=0$ folgt.

Diesen Beweis habe ich in nicht wesentlich anderer Gestalt schon, früher veröffentlicht (Neue Denkschriften der Schweizerischen naturforschenden Gesellschaft von 1848). Er vereinfacht sich etwas, wenn man die Körper $M_{1}$, $M_{2}, \ldots M_{m}$ als homogen betrachtet, da alsdann die Gleichung (1.) die Form

$$
\text { (6.) } \quad \boldsymbol{Q}=-\sum_{1}^{m} k_{\lambda} \int \frac{\partial\left(V_{\lambda}+Q_{\lambda}\right)}{\partial k_{\lambda}} \frac{d w_{\lambda}}{\varrho_{\lambda}}
$$

annimmt.

Herr Kirchhoff machte mich darauf aufmerksam, dafs sich in diesem Falle die Gleichung (5.) einfacher folgendermafsen herleiten läfst. Es ist

$$
\text { (7.) } W=-\sum_{1}^{m} k_{3} \int\left(\frac{\partial W_{\lambda}}{\partial u}\right) \frac{d w_{\lambda}}{\varrho_{k}} \text {; }
$$

woraus, nach einer bekannten Eigenschaft der Oberflächenpotentiale,

$$
\left(\frac{\partial W}{\partial n}\right)^{\prime}-\left(\frac{\partial W}{\partial n}\right)^{\prime \prime}=4 \pi k \frac{\partial W}{\partial n}
$$

folgt, wenn die Werthe von $\left(\frac{\partial W}{\partial n}\right)$ für Puncte unendlich nahe an der Oherfläche, aufserhalb und innerhalb, resp. durch $\left(\frac{\partial W}{\partial n}\right)^{\prime}$ und $\left(\frac{\partial W}{\partial n}\right)^{\prime \prime}$ bezeichnet werden. Also ist auch

$$
\text { (8.) } \sum_{i}^{m} \int W_{\lambda}\left(\frac{\partial W_{\lambda}}{\partial n}\right)^{\prime} d w_{\lambda}=\sum_{i}^{m}\left(1+4 \pi k_{\lambda}\right) \int W_{\lambda}\left(\frac{\partial W_{\lambda}}{\partial n}\right)^{\prime \prime} d w_{\lambda} .
$$

Mit Hülfe der Gleichung ( $\boldsymbol{A}$.) zeigt sich aber leicht, dafs

$-\sum_{1}^{m} \int W_{\lambda}\left(\frac{\partial W_{2}}{\partial n}\right)^{\prime} d w_{\lambda}=\iiint[\boldsymbol{W}, \boldsymbol{W}] d x d y d z$,

$\sum_{1}^{m} \int W_{\lambda}\left(\frac{\partial W_{\lambda}}{\partial n}\right)^{\prime \prime} d w_{\lambda}=\sum_{1}^{m} \iiint\left[W_{k}, W_{k}\right] d x_{\lambda} d y_{\lambda} d z_{\lambda}$ ist. 
Die dreifachen Integrationen in diesen Gleichangen erstrecken sich in der ersten über den ganzen unendlichen Raum au/serhalh der Körper $\boldsymbol{M}_{1}, \boldsymbol{M}_{2}, \ldots$, in der letzten über den Raum innerhalb. Verbindet man sie mit der Gleichung (8.), so erhält man die Gleichung (5.), woraus man wie oben weiter schliefst.

Übrigens läfst sich dieses Verfahren auch, mit einiger Modification; auf die allgemeine Form der Gleichung (2.) ausdehnen.

II.

Einfacher noch läfst sich der analoge Satz in der Theorie der Wärme nachweisen.

Die Temperatur $u$ eines beliebigen Puncts im Imnern eines festen Körpers ist als Function der Zeit $t$ vollkommen bestimmt, wenn folgende Data bekannt sind:

1) Die. Temperatur des Körpers, zur Zeit $t=0$, als Function der Coordinaten, $u_{t=a}=f(x, y, \varepsilon)$.

2) Die Temperatur der Umgebung, als Function

$$
v=\varphi(x, y, z, t)
$$

der Coordinaten der Oberfläche und der Zeit.

3) Die innere Leitungsfähigkeit des Körpers. Diese kann von einem Punct zum andern, und im selben Puncte, nach den verschiedenen Richtungen hin verschieden sein. Sie sei $k_{\mathfrak{1}}$ nach der Richlung der $x$ Axe, $k_{2}$ und $k_{3}$ nach der Richtung der $y$ und $z$ Axe. $k_{1}, k_{2}, k_{3}$ sind als gegebene Functionen von $x, y_{2} z$ zu betrachten. Eben so

4) Die äufsere Leitungsfähigkeit $h$, und

5) Die specifische Wärme $\eta$. Zur Vereinfachung sei dieselbe auf die Einheit des Volumens bezogen, statt, wie gewöhnlich, auf die Einheit der Masse.

Die Temperatur $u$ wird als Function der Coordinaten und der Zeit durch folgende Gleichungen bestimmt:

$$
\text { (1.) } \eta \frac{\partial u}{\partial t}=\frac{\partial\left(k_{1} \frac{\partial u}{\partial x}\right)}{\partial x}+\frac{\partial\left(k_{2} \frac{\partial u}{\partial y}\right)}{\partial y}+\frac{\partial\left(k_{3} \frac{\partial u}{\partial z}\right)}{\partial z},
$$

$$
\begin{gathered}
k_{1} \frac{\partial u}{\partial x} \cos \alpha+k_{2} \frac{\partial u}{\partial y} \cos \beta+k_{3} \frac{\partial u}{\partial z} \cos \gamma+h \psi(u, v)=0 \\
\text { (3.) } u_{t=0}=f\left(x, x_{2} z\right)
\end{gathered}
$$


Die erste dieser Gleichungen gilt für alle Puncte im Innern des Körpers, die zweite für die Puncte der Oberfläche. $\alpha, \beta, \gamma$ haben dieselbe Bedeutung wie oben Die Gröfsen $\eta, k_{1}, k_{2}, k_{3}, h$ sind ihrer Natur nach positiv; aufserdem betrachten wir sie als unabhängig von $u$ und, mit Ausnahme von $h$, als stetige Functionen von $x, y, z$. Die Function $\psi(u, v)$ ist so beschaffen, dafs für $u>u^{\prime}, \psi(u, v)>\psi\left(\boldsymbol{u}^{\prime}, v\right)$, also immer

$$
\text { (4.) } \quad\left(u-u^{\prime}\right)\left(\psi(u, v)-\psi\left(u^{\prime}, v\right)\right) \geqq 0
$$

ist. In der Regel setzt man $\psi(u, v)=u-v$. Wir wollen nun folgenden Satz beweisen.

"Es giebt nur eine Function $u$, welche die Bedingungen (1,2 und 3.) ".gleichzeitig erfüllt."

Beweis. Man setze wieder die Möglichkeit zweier Lösungen $u$ und $\boldsymbol{u}^{\prime}$ des Problems. Macht man $u-u^{\prime}=w$, so erhält man, in Folge der vorstehenden Gleichungen:

$$
\begin{aligned}
& \text { (5.) } \eta \frac{\partial w}{\partial t}=\frac{\partial\left(k_{1} \frac{\partial w}{\partial x}\right)}{\partial x}+\frac{\partial\left(k_{2} \frac{\partial w}{\partial y}\right)}{\partial y}+\frac{\partial\left(k_{3} \frac{\partial w}{\partial z}\right)}{\partial z} \\
& \text { (6.) } k_{1} \frac{\partial w}{\partial x} \cos \alpha+k_{2} \frac{\partial w}{\partial y} \cos \beta+k_{3} \frac{\partial w}{\partial z} \cos \gamma+h \varphi=0,
\end{aligned}
$$

wo zur Abkürzung

$$
\varphi=\psi(u, v)-\psi\left(u^{\prime}, v\right)
$$

gesetzt ist. Die erste Gleichung gilt für alle Puncte im Innern, die zweite für Puncte der Oberfläche. Aufserdem hat man für alle Puncte im Innern:

$$
\text { (7.) } \quad w=0 \text { für } t=0 \text {. }
$$

Macht man in der Gleichung (B.), $U=U^{\prime}=w$, und wendet die Gleichungen (5.) und (6.) an, so ergiebt sich

$$
=\iiint \eta w \frac{\partial w}{\partial t} d x d y d z+\iiint\left\{k_{1}\left(\frac{\partial w}{\partial x}\right)^{2}+k_{2}\left(\frac{\partial w}{\partial y}\right)^{2}+k_{3}\left(\frac{\partial w}{\partial z}\right)^{2}\right\} d x d y d z
$$

oder, wenn man $\eta$ als von $t$ unabhängig betrachtet:

(8.) $\frac{1}{2} \frac{\partial p}{\partial t}=-\left\{\int h w \varphi d w+\iiint\left(k_{1}\left(\frac{\partial w}{\partial x}\right)^{2}+k_{2}\left(\frac{\partial w}{\partial y}\right)^{2}+k_{3}\left(\frac{\partial w}{\partial z}\right)^{2}\right) d x d y d z\right\}$, wo $p=\iiint \eta w^{2} d x d y d z$ ist.

Der in der Parenthese enthaltene Ausdruck ist positiv, wenn $u$ und $\boldsymbol{u}^{\prime}$, also auch $w$ reell ist. Denn nach (4.) ist hw eine positive Gröfse und das letzte Integral in (8.) besteht aus einer Summe reeller Quadrate, multiplicirt 
mit positiven Coëfficienten. Die Seite rechts der Gleichung (8.), d. h. der Differentialquotient von $p$, nach $t$ genommen, ist daher negativ, wenn er von 0 verschieden ist, welches im übrigen auch der Werth von $w$ sein mag. Hieraus folgt, dafs wenn $t$ wächst, die Function $p$ immer abnehmen mufs, wenigstens nie zunehmen kann. Aber für $t=0$ ist $w=0$, für alle Werthe von $x, y, z$, über welche die Integrationen in der Gleichung (8.) ausgedehnt sind; also ist $p=0$. Da aber $p$ mit wachsendem $t$ nicht zunehmen und, als Summe von reellen Quadraten, multiplicirt mit positiven Coëfficienten, auch nicht negativ werden kann, so folgt, dafs für jeden Werth von $t, p=0$ sein mufs. Aber aus

$$
p=\int \eta w^{2} d x d y d z=0
$$

folgt, dafs identisch $w=0$, also $u=u^{\prime}$ ist. Das Problem läfst also nur eine Lösung zu.

Ganz wie in der vorigen Nr. läfst sich nun weiter zeigen, dafs auch kein von 0 verschiedener imaginärer Ausdruck von $w$ den Gleichungen $(5,6,7$. gleichzeitig genügt.

In der Theorie der Wärme wendet man zuweilen den Satz an, dafs, wenn $v$, wit wachsendem $t$, sich einer constanten Grenze $v_{0}$ nähert, $v_{0}$ ebenfalls die Grenze von $u$ ist, für $t=\infty$. Ich glaube nicht, dafs bis jetzt ein Beweis dieses Satzes gegeben worden ist; man begnügte sich, einen Erfahrungssatz der Physik auf ein rein analytisches Problem zu übertragen. Der strenge Beweis ergiebt sich aber sehr leicht mit Hülfe der Gleichung (8.). Es sei z. B. von Anfang an $v=v_{0} \doteq$ Const., so werden offenbar die Gleichungen (1 und 2.) erfüllt, wenn man $u=v_{0}$ setzt. Es sei ferner $u^{\prime}$ die Function, welche den Gleichungen $(1,2,3$.) gleichzeitig genügt. Offenbar werden die Gleichungen (5 und 6.), also auch die Gleichung (8.) erfüllt, wenn man $w=v_{0}-u^{\prime}$ setzt. Da nun der Differentialquotient von $p=\frac{1}{2} \int \eta\left(v_{0}-u^{\prime}\right)^{2} d x d y d z$, nach $t$ genommen, beständig negativ ist, so lange $w$ endlich ist, so folgt, dafs sich für $t=\infty, w=\left(v_{0}-u^{\prime}\right)$ der Grenze 0 für alle Werthe von $x, y, z$ nähern mufs; d. h. es wird $u_{t=\infty}=v_{0}$.

Die hier angestellten Betrachtungen lassen sich leicht verallgemeinern. Man kann z. B. setzen, dafs $k_{1}, k_{2}, k_{3}, h, \eta$ Functionen von $t$ und selbst von $u$ seien. Diese Functionen sind aher gewissen Bedingungen unterworfen, wenn der bewiesene Satz gelten soll. Man kann ferner annehmen, dafs statt eines einzigen Körpers ein System von Körpern gegeben ist, mit belieb́igen Anfangstemperaturen, und dafs sie in einem diathermalen Mittel von beliebig 
veränderlicher Temperatur, der gegenseitigen Einwirkung, theils durch Strahlung, theils in Folge von Berührung ausgesetzt sind. Sind die Gröfsen $k$ oder $\eta$ längs einer Fläche discontinuirlich, so findet für die Puncle derselben eine Gleichung von der Form (2.) Statt.

Dafs das Problem des Gleichgewichts der Temperaturen nur eine Lösung zuläfst, folgt als Corollar aus dem Vorigen. Der selbständige Beweis liefse sich auf die Gleichung

$$
0=\int \boldsymbol{h} w \varphi d w+\iiint\left\{k_{1}\left(\frac{\partial w}{\partial x}\right)^{2}+k_{2}\left(\frac{\partial w}{\partial y}\right)^{2}+k_{3}\left(\frac{\partial w}{\partial z}\right)\right\} d x d y d s
$$

gründen; aus welcher folgt, dafs für alle Puncte im Innern:

$$
\frac{\partial w}{\partial x}=0, \quad \frac{\partial w}{\partial y}=0, \quad \frac{\partial w}{\partial z}=0,
$$

also $w=$ Const. ist. Aufserdem folgt $w=0$ für alle Puncte der Oberfläche, also, da $w$ eine stetige Function ist, Const. $=0$, oder $u=u^{\prime}$.

III.

Man setze, es sei in der Gleichung (2.) der vorigen $\mathrm{Nr} . \psi(u, v)=u-v$. Bekanntlich kann die Bestimmung der Function $u$, welche den Gleichungen $(1,2,3$.$) genůgt, auf die Bestimmung einer andern Function U$ zurückgeführt werden, welche folgenden Gleichungen genügt:

$$
\begin{gathered}
\text { (1.) } \eta \frac{\partial U}{\partial t}=\frac{\partial\left(k_{1} \frac{\partial U}{\partial x}\right)}{\partial x}+\frac{\partial\left(k_{2} \frac{\partial U}{\partial y}\right)}{\partial y}+\frac{\partial\left(k_{3} \frac{\partial U}{\partial z}\right)}{\partial z} \\
\text { (2.) } k_{1} \frac{\partial U}{\partial x} \cos \alpha+k_{2} \frac{\partial U}{\partial y} \cos \beta+k_{3} \frac{\partial U}{\partial z} \cos \gamma+h U=0 . \\
\text { (3.) } U_{t=0}=F(x, y, z) .
\end{gathered}
$$

Die erste Gleichung gilt für Puncte im Innern, die zweite für Puncte der Oberfläche. Beiden kann gleichzeitig durch einen Ausdruck von der Form

$$
\boldsymbol{a} \boldsymbol{P} e^{-\mu t}
$$

genügt werden. $a$ ist eine willkürliche Constante, $P$ findet sich aus der partiellen Differentialgleichung

$$
\text { (4.) }-\mu \eta \boldsymbol{P}=\frac{\partial\left(k_{1} \frac{\partial \boldsymbol{P}}{\partial x}\right)}{\partial x}+\frac{\partial\left(k_{2} \frac{\partial \boldsymbol{P}}{\partial y}\right)}{\partial y}+\frac{\partial\left(k_{3} \frac{\partial \boldsymbol{P}}{\partial z}\right)}{\partial z} \text {. }
$$

Die Constante $\mu$ ist eine Wurzel der Gleichung

$$
k_{1} \frac{\partial \boldsymbol{P}}{\partial x} \cos \alpha+k_{2} \frac{\partial \boldsymbol{P}}{\partial y} \cos \beta+k_{3} \frac{\partial \boldsymbol{P}}{\partial z} \cos \gamma+h \boldsymbol{P}=0 \text {. }
$$


Diese Gleichung ist im allgemeinen transcendent und hat unendlich viele Wurzeln. Bezeichnet man dieselbén durch $\mu_{1}, \mu_{2}, \ldots \mu_{m}, \ldots$ und deutet die von ihnen abhängigen Grōfsen durch die nämlichen Indices an, so hat die allgemeinste Function, welche den Gleichungen (1 und 2.) genügt, die Form

(6). $\quad \boldsymbol{U}=\dot{\Sigma} \boldsymbol{a}_{m} \boldsymbol{P}_{m} \boldsymbol{e}^{-\mu m t}$.

Die Constanten $a$ sind so zu bestimmen, dafs die Gleichung (3.) erfüllt wird, d. h. so, dafs für alle Puncte im Innern des Körpers,

$$
\text { (7.) } \quad \boldsymbol{F}(x, y, z)=\sum \boldsymbol{u}_{m} \boldsymbol{P}_{m}
$$

ist. Die Möglichkeit, auf diesem Wege die Aufgabe zu lösen, hangt von folgenden Bedingungen ab.

1) Für $t=\infty$ darf $U$ nicht unendlich und auch zu keiner periodischen Function von $t$ werden. Denn nach der vorigen $\mathrm{Nr}$. mufs sogar $\boldsymbol{U}_{t=\infty}=0$ sein. Es ist also nöthig, dafs die Gleichung (5.) reelle und positive Wurzeln habe. Falls es imaginäre oder negative Wurzeln giebt, müssen die davon abhängigen Glieder aus dem Ausdruck (6.) wegfallen.

2) Die Lösung der allgemeinen Aufgabe setzt voraus, dafs eine ganz willkürlich gegebene Function $\boldsymbol{F}(x, y, z)$ sich innerhalb bestimmter Grenzen in eine Reihe von der Form (7.) entwickeln lasse. Hiezu ist nöthig, dafs die Reihe unendlich viele Glieder, also die Gleichung (5.) unendlich viele reelle und positive Wurzeln habe. Dafs Dieses auch genügend sei, mufs besonders nachgewiesen werden.

Poisson zeigt, dafs alle Wurzeln der Gleichung (5.) reell sind. (Théor. math. de la chaleur, p. 178.) Dafs sie auch positiv sind, weiset er nur in besonderen Fällen aus der Form der Gleichung nach. (Ibid. p. 294.) Poisson geht von der Gleichung

$$
\iint \eta P_{1} d x d y d z=0
$$

aus, in welcher $\boldsymbol{P}$ und $\boldsymbol{P}_{1}$ den Wurzeln $\mu=\lambda^{2}$ und $\mu_{1}=\lambda_{1}^{2}$ entsprechen. Diese Gleichung gilt aber nur, wenn $\lambda^{2}$ und $\eta \lambda_{1}^{2}$ verschieden sind. Nemlich es ist allgemein $\left(\lambda^{2}-\lambda_{1}^{2}\right) \iiint^{0} \boldsymbol{P P} P_{1} d x d y d z=0$. Setzt man nun $\lambda=g+g^{\prime} \gamma-1$, $\lambda_{1}=g-g^{\prime} \gamma^{\prime}-1$, so folgt, dafs

$$
2 g y^{\prime} \sqrt{ }-1 \iiint\left(G^{2}+G^{\prime 2}\right) \eta d x d y d z=0
$$

sein mufs. Hieraus folgt aber nur, dal's $g g^{\prime}=0$ sein, d. h., dal's $\lambda^{2}$ reell sein mufs; dafs $g^{\prime}=0$, folglich $\lambda^{2}$ positiv sein mufs, folgt auf diesem Wege nicht. Indessen kann der Beweis auch allgemein gegeben werden. 
Es seien nemlich $\mu$ und $\mu_{1}$ zwei Wurzeln der Gleichung (5.), und $\boldsymbol{P}$ und $\boldsymbol{P}_{1}$ die entsprechenden Integrale der Gleichung (4.). In der Gleichung (B.) setze man

$$
\boldsymbol{U}=\boldsymbol{e}^{-\mu t} \boldsymbol{P}, \quad \boldsymbol{U}^{\prime}=\boldsymbol{P},
$$

so erhält man, wenn man den Factor $e^{-\mu t}$ wegläfst und die Gleichungen (4 und 5.) anwendet:

$$
\begin{aligned}
& \quad 0=-\mu \iiint \eta P_{1} d x d y d z \\
& +\iiint\left\{k_{1} \frac{\partial P}{\partial x} \cdot \frac{\partial P_{1}}{\partial x}+k_{2} \frac{\partial P}{\partial y} \cdot \frac{\partial P_{1}}{\partial y}+k_{3} \frac{\partial P}{\partial z} \cdot \frac{\partial P_{1}}{\partial z}\right\} d x d y d z+\int h P_{1} d w
\end{aligned}
$$

Nun nehme man an, die Gleichung (5.) habe eine Wurzel von der Form $\mu=\mu^{\prime}+\mu^{\prime \prime} \gamma-1$, wo $\mu^{\prime}$ und $\mu^{\prime \prime}$ reell sind, so müfste, da sich $\boldsymbol{P}$ als reelle Function von $\mu, x, y, z$ voraussetzen läfst, eine zweite Wurzel von der Form $\mu_{1}=\mu^{\prime}-\mu \sqrt{ }-1$ vorkommen. Diese Werthe in $\boldsymbol{P}$ und $\boldsymbol{P}_{1}$ substituirt, würden

$$
\boldsymbol{P}=\boldsymbol{G}+\boldsymbol{G}^{\prime} \sqrt{ }-1, \quad \boldsymbol{P}_{1}=\boldsymbol{G}-\boldsymbol{G}^{\prime} \sqrt{ }-1
$$

geben, und dadurch geht die Gleichung (8.) in

$$
\begin{aligned}
\mathbf{0}= & -\left(\mu^{\prime}+\mu^{\prime \prime} \sqrt{ }-1\right) \iiint \eta\left(G^{2}+G^{2}\right) d x d y d z+\int\left(G^{2}+G^{\prime 2}\right) h d w \\
& +\iiint\left\{k_{1}\left(\left(\frac{\partial G}{\partial x}\right)^{2}+\left(\frac{\partial G^{\prime}}{\partial x}\right)^{2}\right)+k_{2}\left(\left(\frac{\partial G}{\partial y}\right)^{2}+\left(\frac{\partial G^{\prime}}{\partial y}\right)^{2}\right)\right. \\
& \left.+k_{3}\left(\left(\frac{\partial G}{\partial z}\right)^{2}+\left(\frac{\partial G^{\prime}}{\partial z}\right)^{2}\right)\right\} d x d y d z
\end{aligned}
$$

über. In dieser Gleichung sind alle Glieder reell, aufser den in $\mu^{\prime \prime}$ multiplicirten. Diese also müssen besonders verschwinden; d. h. es mufs

$$
0=\sqrt{-1} \mu^{\prime \prime} \iiint \eta\left(G^{2}+G^{\prime 2}\right) d x d y d z,
$$

also $\mu^{\prime \prime}=0, \mu=\mu_{1}$ und folglich $G^{\prime}=0, P=P_{1}=G$ sein. Die obige Gleichung wird demnach $\mathrm{zu}$

$$
\begin{aligned}
0= & -\mu \iiint \eta \boldsymbol{P}^{2} d x d y d z+\int \boldsymbol{P}^{2} h d w \\
& +\iiint\left\{k_{1}\left(\frac{\partial P}{\partial x}\right)^{2}+k_{2}\left(\frac{\partial P}{\partial y}\right)^{2}+k_{3}\left(\frac{\partial P}{\partial z}\right)^{2}\right\} d x d y d z .
\end{aligned}
$$

Diese Gleichung kann aber offenbar nur bestehen, wenn $\mu$ positiv ist. Denn wäre $\mu$ negativ, so bestände ihr zweites Glied aus einer Summe reeller und positiver Gröfsen, und könnte also nicht verschwinden. Alle Wurzeln der Gleichung (5.) müssen also reell und positiv sein.

Zürich, den 5ten Januar 1851. 